\title{
The effect of pressure on high- and low-working-memory students: An elaboration of the choking under pressure hypothesis
}

\author{
Zuowei Wang ${ }^{\prime *}$ and Priti Shah ${ }^{2}$ \\ 'Combined Program in Education and Psychology, University of Michigan, Ann Arbor, \\ Michigan, USA \\ ${ }^{2}$ Department of Psychology, University of Michigan, Ann Arbor, Michigan, USA
}

Sample. Fifty-three third and fourth graders from China participated in this study.

Method. Participants' working memory (WM) was assessed by the Automated Operation Span task. Then, they solved mental addition problems of different types under low- and high-pressure conditions. Performance was analysed as a function of pressure condition, working memory capacity, and problem type.

Results. On 'no carry' mental addition problems, there was no difference between the two groups of children regardless the presence of pressure. For problems with carries, low WM (LWM) children performed worse on all tasks compared with high WM (HWM) children in the no-pressure condition, but pressure influenced the LWM and HWM differently depending on the nature of the carrying task. On 'hidden carry' mental addition trials (for which guessing strategies were minimally effective), LWM performance was much lower than HWM performance under pressure. By contrast, performance was similar between LWM and HWM groups under pressure on the 'normal carry' trials that allowed for non-resource-intensive heuristic strategies.

Conclusion. Whether high- or low-working-memory elementary-school-aged children were more or less affected by pressure was dependent on task-difficulty and the types of strategies that could be used to solve the problems.

Situations in which performing at one's best is important - the final round of a tennis tournament, the million dollar question in a game show, a high stakes college entrance exam - are all situations that can lead to decrements that are attributable to the pressure of the situation (Baumeister, 1984). This choking under pressure phenomenon is important to understand because of the importance of these contexts. One unfortunate fact about choking under pressure is that individuals with high ability are frequently found to choke under pressure more so than those with low ability (Beilock, 2008; Beilock \& Carr, 2005; Gimmig, Huguet, Caverni, \& Cury, 2006). That individuals may be affected by pressure to different degrees reduces the validity of competition in all domains. The goal of the present study is to test a hypothesis about the conditions under which higher ability individuals may be more likely to choke, and the conditions under which lower ability individuals may be more likely to choke.

*Correspondence should be addressed to Zuowei Wang, 610 E. University Ave, Room 1406, University of Michigan, Ann Arbor, MI 48109, USA (email: zwwang@umich.edu). 
Research on choking on cognitive tasks has focused on tasks that are demanding of working memory, the cognitive system responsible for the active maintenance of task-relevant information (Jonides, 1995; Jonides et al., 2008; Shah \& Miyake, 1999). The primary assumption of these studies is that dealing with pressure itself is demanding of working memory resources that are known to be highly limited. Specifically, pressure is assumed to lead to worry about performance and other off-task thoughts, and these off-task thoughts consume working memory. In the well-known study (Beilock \& Carr, 2005), for example, individuals with high working memory (HWM) and low working memory (LWM) were asked to perform modular arithmetic tasks in either a low-pressure or a high-pressure context. Such modular arithmetic tasks can be performed by computation (which is highly-working-memory demanding) or by estimation (which requires fewer working memory resources but does not always yield the correct response). The authors found that the HWM participants were more affected by the pressure than the LWM participants. They explained their results by arguing that HWM individuals were more likely to use the more resource-intensive computational approaches than the LWM individuals; thus, any working memory demands such as attention to off-task thoughts generated in the pressure situation had more effect on these participants. In a related study, Gimmig et al. (2006) also found that HWM individuals were more likely to choke under pressure than LWM individuals when performing fluid reasoning tasks. They provided an alternative explanation for the high-ability choking under pressure phenomenon: HWM participants may be more concerned about their performance; thus, the pressure context leads to even more off-tasks thoughts than it does for LWM individuals.

Studies of math anxiety suggest that trait anxiety, such as pressure, also reduces the effective working memory capacity of individuals (Ashcraft \& Kirk, 2001; Ashcraft \& Krause, 2007). Ashcraft and Kirk (2001), for example, found that individuals with a higher math anxiety showed less working memory capacity, and they performed much worse when the task became difficult. This suggests that anxiety consumes people's working memory capacity thus compromising performance. However, trait anxiety may not affect performance in the same way as state anxiety (pressure). Johnson and Gronlund (2009) studied the effect of trait anxiety on participants' performance in a dual-task context that combined a tone discrimination task and a short-term memory task. They found an interaction such that LWM participants were more affected by trait anxiety than HWM participants. Are there contexts in which LWM participants are more affected by pressure than HWM participants just as they are sometimes more affected by trait anxiety?

One approach to clarify findings in this domain may be to consider the role of task-difficulty, the nature of the tasks (those that allow for multiple strategies vs. those that do not), and the type of pressure manipulation used in the different studies. First, it is possible that HWM individuals, when performing a task that is moderately easy for them, have enough resources available to them even if some of their attention is diverted by a pressure context. LWM individuals may be more affected on these easy tasks because they are, without pressure, closer to reaching their capacity limits. Thus, on easier tasks, it may appear that LWM individuals choke more than HWM individuals. On more difficult tasks, HWM individuals may be close to their own capacity limits and thus may be affected by pressure, whereas LWM individuals may use guessing strategies or other heuristics that yield above chance performance but are not WM demanding. Indeed, the earlier work on choking under pressure finds this exact pattern. HWM individuals in the Beilock and Carr (2005) study were most affected by pressure on the difficult modular arithmetic problems. In a later study, Beilock and DeCaro (2007) provided evidence that LWMs were using 


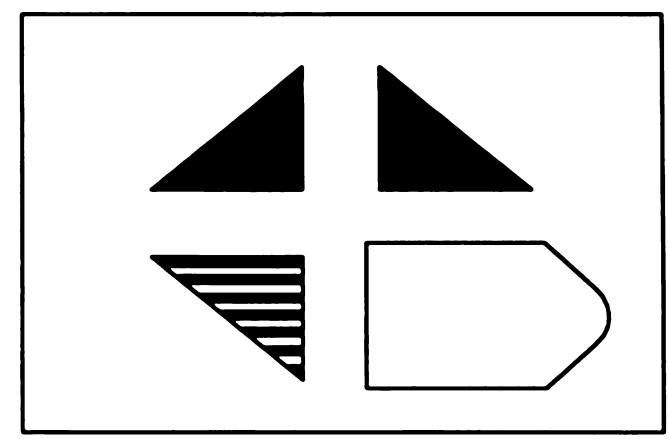

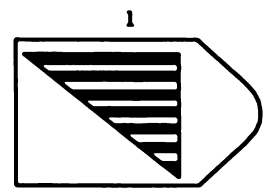

4

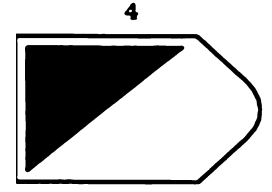

2

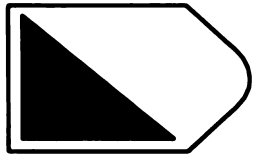

5

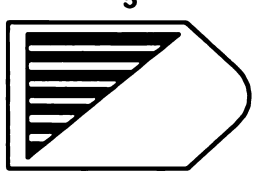

3

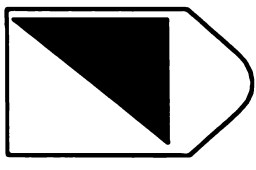

6

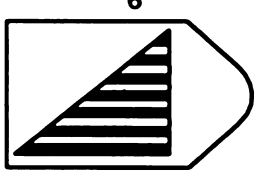

Figure I. Example of matrix reasoning problem.

estimation instead of working-memory-intensive calculation, and some of the HWMs also switched their strategy from using a working-memory-intensive strategy to using estimation when they were under pressure. The Raven's Standard Progressive Matrices task, used in the Gimmig et al. (2006) study, is also highly working memory demanding but allows for the use of less demanding heuristics. Consider, for example, the sample matrix reasoning problem in Figure 1. A participant may be able to easily eliminate the solid coloured options and thus has a 33\% chance of identifying the correct response by guessing. If HWM individuals rely on computational strategies that are working memory demanding, whereas LWM individuals rely on heuristics and guessing, what looks like not choking under pressure may be due to a pseudo-floor effect. The paradigm used by Johnson and Gronlund (2009), however, may be less amenable to successful use of LWM demanding heuristic strategies because of their dual-task nature. Thus, it is not surprising that in this case, the LWMs (who had to rely on working memory resources to perform the dual task) are more affected by pressure than HWM individuals (who, presumably, had more capacity to spare to deal with the stress).

Additionally, it is not clear whether the pressure manipulation in previous studies was equally effective for HWM and LWM individuals. If, for example, HWM individuals feel greater desire to succeed in cognitive tasks because of past performance and motivation, then a competitive pressure manipulation such as those used in the Beilock and Carr (2005) and Gimmig et al. (2006) studies may lead to more off-task thoughts. Thus, the pressure manipulation may actually consume more working memory resources in HWM individuals. LWM individuals, by contrast, may not expect to do well or have a stake in good performance. Thus, the pressure manipulation may have been unimportant to them. Indeed, the Gimmig et al. (2006) study found that HWM individuals were more likely to feel anxiety with a pressure manipulation than LWM individuals. On the other hand, 
the trait anxiety studies included both high and LWM individuals and no experimental pressure manipulation. Thus, these studies implicitly control for possible differences in effects of pressure by generating high anxiety in both LWM and HWM participants.

To sum up, we predict that pressure will decrease individual performance if (1) the task is challenging enough that successful performance requires the individual to use most of their resources on the task at hand, (2) the individual in the baseline condition performed the task using a working memory demanding strategy rather than heuristic strategies (often HWM individuals), and (3) the pressure manipulation was effective.

We consider the impact of pressure on the performance of high- and low-working-memory individuals on different task types within a single study. By manipulating the working memory demands and potentially effective strategies of the same task, we can make comparisons across conditions within the same study. Moreover, instead of using abstract laboratory tasks, we attempt to replicate previous findings by implementing real-life classroom tasks. Most of the previous research on pressure and working memory capacity used tasks that were unfamiliar to participants. It is possible that the effects of pressure may be different in familiar contexts, and it is important to assess how pressure may affect performance outside the laboratory.

In the current study, we recruited third and fourth graders in China and asked them to perform 3-digit mental addition problems under no pressure or high pressure. We modified Beilock and Carr's (2005) procedure to generate a high-pressure but noncompetitive context: children were tested individually with an experimenter watching, and they were reminded the importance of the test. In addition, camera was placed behind them when they were taking the test. Finally, they were told that education experts in the United States would evaluate their performance.

We selected mental addition because it is a common activity for third- and fourthgrade students in China and has general educational relevance. Much of the work on pressure and anxiety on performance has focused on mathematics contexts, and high stakes testing typically includes a mathematical component. Furthermore, this task allows us to manipulate systematically the working memory demands and potentially successful problem-solving strategies.

In the school where we performed the experiment, children are encouraged to perform multidigit addition and subtraction in their 'heads' without the aid of paper and pencil. Children's mental arithmetic skill is also appreciated in the general Chinese culture (Stigler, 1984). Previous studies have used tasks that are rather unfamiliar and not commonly introduced in classroom contexts, such as modular arithmetic (Beilock \& Carr, 2005) and tone discrimination (Johnson \& Gronlund, 2009). It is possible that some of the previous findings are dependent on tasks being unfamiliar, and pressure has less impact on common, everyday tasks. Using mental addition task allows us to investigate how big an impact pressure may have in more familiar, well-practised contexts. It also provides an opportunity for addressing the effect of performance pressure in a different cultural setting than previous studies on this topic.

Previous studies have demonstrated that working memory is important in the performance of mental arithmetic tasks (Hitch, 1978). By manipulating the number of 'carries', it is possible to manipulate the working memory demands of mental addition. This is another advantage of the mental arithmetic task used in the study. We asked children to solve 3-digit addition problems from left to right (hundreds digit to unit digit), because this is the way they learned mental addition in school.

In this study, problems were presented on a screen, and then participants were asked to select the correct answer from two options that were presented on the next screen. 
One of the options was the correct answer, and for the lure option, a single digit in the sum was altered by 1 . The number of carries varied from 0 to 3 and examples of each type are shown in Figure 2. Zero-carry problems are ones in which the sum of each column is $<10$, thus requiring no 'carrying' between the hundreds, tens, and unit columns (Figure 2a). We predicted that zero-carry problems have such low WM demands that performance would not be disrupted by pressure.

The 1-carry problems have a carry only in one of the three columns (Figure 2b), and the 2-carry problems have a carry in two of the three columns (Figure 2c). The 3-carry problems always have a carry in all three columns (resulting in an answer above 1,000, Figure $2 \mathrm{~d}$ ). These three types of problems are grouped together in the analysis to form a difficulty gradient so that neither ceiling effect nor floor effect could occur, which provide room for the performance change under pressure. We classified these problems as 'normal carry' problems. Although the calculation of these problems could be highly working memory demanding, subjects who are not able to perform complete calculation are given a suboptimal 'backdoor' to get to the correct option. That is, although they may only be able to calculate the results at one or two columns, if the column(s) that they remember include the lure digit, then they can easily distinguish between the lure and correct response, thus performing well above chance. ${ }^{1}$ It should also be noted that compared with HWM children, LWM children are more likely to use this backdoor due to their insufficient working memory. Moreover, using the backdoor strategy is less subject to the influence of pressure because the working memory demand is low for this strategy. Thus, we predict that for these normal carry problems, LWM students may be less affected by pressure than HWM students.

Finally, a third type of problem was designed to shut the backdoor, which we call 'hidden carry' problem (Figure 2e). The hidden carry problem literally has 3 carries, but the sum of the ten's column is 9 and the carry at this digit is only apparent when the unit's digit is calculated and put a carry to the ten's digit, thus turning the 9 here into 10 .

'Probability of getting the correct answer for those who are not doing complete calculation in the normal carry problems: (I). If a subject only maintains the calculation result at one column:

$p$ (the column is just where the correct and lure options differ) $=1 / 3$

$\rightarrow$ get the correct answer.

$p$ (the column is other than where the correct and lure options differ) $=2 / 3$

$\rightarrow$ do not know which option is correct, so randomly pick one.

$p$ (picking the correct answer from the two options by guessing) $=1 / 2$

In sum, the odd that a subject who only maintains the calculation result at one column gets the correct answer is:

$$
\frac{1}{3}+\frac{2}{3} \times \frac{1}{2}=0.67
$$

(2). If a subject only maintains the calculation result at two columns:

$p$ (the column where the correct and lure options differ is among the maintained column) $=2 / 3$

$\rightarrow$ get the correct answer.

$p$ (the column where the correct and lure options differ is not among the maintained column) $=1 / 3$

$\rightarrow$ do not know which one is correct, so randomly pick one.

$p$ (picking the correct answer from the two options by guessing) $=1 / 2$

In sum, the odd that a subject who only maintains the calculation result at two columns gets the correct answer is:

$$
\frac{2}{3}+\frac{1}{3} \times \frac{1}{2}=0.83
$$


(a)

(b)

(c)

(d)

(e)

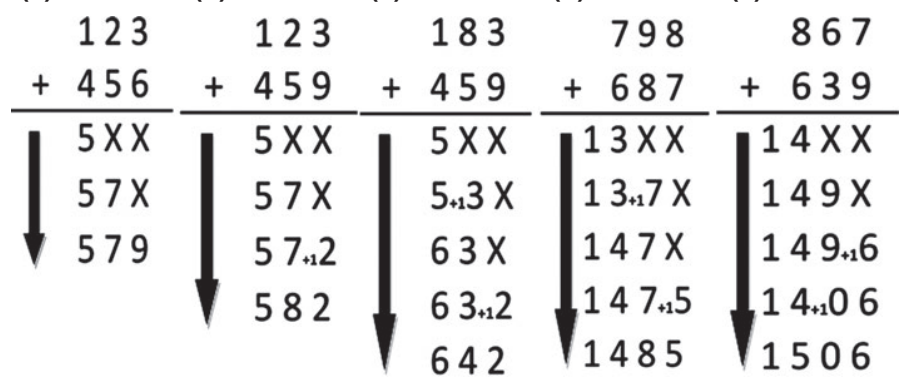

Figure 2. Illustration of problem solving procedures. (Panel a) No carry; (Panels b-d) normal carry, number of carries varies from I to 3; (Panel e) hidden carry (at the tens digit).

This setting of the problem shuts the backdoor: If subjects fail to perform complete calculation, due to the hidden carry, the partial calculation will give them exactly the result in the lure option. Thus, the guessing rate is no longer higher than $50 \%$, and WM reduction should be highly disruptive. In this case, as all participants must use computational-intensive strategies, LWM individuals should be more affected than HWM individuals.

In summary, the study has three hypotheses. First, for easy mental addition tasks, where no carry was required and working memory requirement was the least intensive, pressure should minimally affect HWMs' and LWMs' performance. Second, for the normal carry condition, we would replicate Beilock and Carr (2005) findings. HWMs' advantage would decrease in the pressure condition, because pressure consumes HWMs' necessary cognition resources for their advantage, whereas this does not happen in LWMs, because the task could already be too hard for them when there is no pressure and thus they may be likely to resort to less demanding heuristics. Third, hidden carry condition, we would see the performance difference between HWMs and LWMs magnified by pressure, because the problems in this condition require working memory and failure to use working memory will significantly decrease accuracy.

\section{Method}

\section{Participants}

Fifty-three third- and fourth-grade children from Muling Shiyan Elementary School (Muling, China) were tested. Four additional children were tested, but their data not analysed because their error rate on the operation task in the working memory test was higher than $20 \%$, indicating that they might not be following the instructions of the working memory test (Unsworth, Heitz, Schrock, \& Engle, 2005).

\section{Design}

We used a $2 \times 2 \times 3$ mixed design $(H W M$ vs. $L W M \times$ no pressure vs. pressure $\times$ no carry, normal carry, vs. hidden carry problems).

\section{Materials and procedure}

On day 1, participants first performed the Chinese version of the Automatic Operation Span Task (Aospan; Unsworth et al., 2005) to assess working memory. The task was 
presented to students in groups of approximately 15 children. In this working memory test, participants are asked to solve a set of mathematical equation (e.g., $(9-6) \times 3=$ ?) and then asked to select from two possible alternatives of the answer. Following the presentation of each equation and answer options, they are shown a letter to remember. Then, a new equation and letter combination are presented. There are a total of 2 7 equation per letter items in a trial. At the end of a trial, participants are asked to recall the letters in the order presented. Although this task, on the surface, requires mathematics, individual differences on this task are thought to measure domain-general working memory resources. This test was used because of a readily available version in Chinese.

Each child was tested twice in 2 days. Their mean Aospan score was used to assign them into low- or high-working-memory group (HWM/LWM) with a median split in the following analysis. It should be noted, given that the letters used in the span task were English alphabetic letters, that all children were familiar with the English alphabet and computer keyboard.

Following the first presentation of the span task, participants performed the mental addition task under the no-pressure condition. First, participants performed a practice ${ }^{2}$ block of mental addition trials in which the problem and the two options were on the same screen. After the practice block, children were asked to confirm that they understood the procedure of the task before they went on to the second block. The second block was the same as the first block, except that the options were on a separate screen following the problem screen increasing the working memory demand and constraining the potential strategies that might be used. Both the practice block and the real-task block contained 25 trials. The practice block took 3-4 min; the real-task block took 4-5 min. Performance on the second block was analysed.

The mental addition task required children to add two 3-digit numbers in their mind. Children were asked to try to solve the addition problems as quickly as possible without sacrificing accuracy. On each trial, the two 3-digit numbers were presented vertically on the screen. Children needed to click the mouse after they got the answer. When they clicked the mouse, a new screen containing two options would appear, prompting them to click on the correct option. The lure option differed from the correct option in one of the three digits, that is, its 1, 10, or 100 larger or smaller than the correct option. After children clicked on one of the two options, a feedback screen indicated whether the answer was correct. As mentioned in the introduction, the difficulty level of the mental addition task was manipulated by varying the number of carries of the calculation. There were five no carry, five one-carry, five two-carry, and five three-carry problems (Figure 2a2d), together with five hidden carry problems (Figure 2e, whether the tens digit needs a carry or not depends on the result of the unit digit).

The pressure manipulation was achieved by a contrast between the conditions in the two testing contexts. On the first day when children were doing the tasks without pressure, they were tested in groups of about 15 students together in the student computer laboratory. On the second day, pressure was added by the following manipulations: The children were tested one by one in the teacher computer laboratory; the children were reminded that the first day's testing had been just for practice and the current day's testing was the real test; the first author sat beside the child when he/she was

\footnotetext{
${ }^{2}$ In this block, children were always able to compare their calculation with the two options before they got the result, so they did not have to keep intermediate calculation results in their mind. In other words, the WM demand is low in this block. To control for the potential influence of this practice block on the formal block, children performed this practice block on both days.
} 
Table I. Accuracy data for all the conditions; numbers in parentheses are standard error of the mean

\begin{tabular}{lcclll}
\hline & \multicolumn{2}{c}{ Low pressure } & & \multicolumn{2}{c}{ High pressure } \\
\cline { 2 - 3 } & $\begin{array}{c}\text { Low working } \\
\text { memory group }\end{array}$ & $\begin{array}{c}\text { High working } \\
\text { memory group }\end{array}$ & & $\begin{array}{c}\text { Low working } \\
\text { memory group }\end{array}$ & $\begin{array}{c}\text { High working } \\
\text { memory group }\end{array}$ \\
\hline No carry & $0.835(0.032)$ & $0.885(0.030)$ & & $0.896(0.030)$ & $0.885(0.028)$ \\
Normal carry & $0.707(0.035)$ & $0.836(0.033)$ & & $0.762(0.031)$ & $0.774(0.029)$ \\
Hidden carry & $0.765(0.034)$ & $0.823(0.032)$ & & $0.704(0.037)$ & $0.854(0.035)$ \\
\hline
\end{tabular}

Table 2. Response time data (in milliseconds) for all the conditions; numbers in parentheses are standard error of the mean

\begin{tabular}{lrrrrrr}
\hline & \multicolumn{2}{c}{ Low pressure } & & \multicolumn{2}{c}{ High pressure } \\
\cline { 2 - 3 } & $\begin{array}{c}\text { Low working } \\
\text { memory group }\end{array}$ & $\begin{array}{c}\text { High working } \\
\text { memory group }\end{array}$ & & $\begin{array}{c}\text { Low working } \\
\text { memory group }\end{array}$ & $\begin{array}{c}\text { High working } \\
\text { memory group }\end{array}$ \\
\hline No carry & $6,064(602)$ & $5,412(415)$ & & $6,272(564)$ & $5,006(364)$ \\
Normal carry & $10,309(1,089)$ & $10,556(882)$ & & $10,067(1,121)$ & $8,515(765)$ \\
Hidden carry & $10,459(1,178)$ & $10,446(686)$ & & $9,656(1,168)$ & $8,476(704)$ \\
\hline
\end{tabular}

doing the tasks; there was a video camera placed behind the child's seat; the children were reminded that their performance data on that day were to be taken to the United States and evaluated by education experts. ${ }^{3}$

\section{Results}

We conducted a three-way ANOVA to assess the effect of working memory capacity (HWM/LWM, respectively), problem type (no carry, normal carry, and hidden carry), and pressure (no-pressure, pressure) on mental addition performance (accuracy and RT means for all conditions are presented in Tables 1 and 2, respectively). We found a significant three-way interaction of working memory capacity $\times$ problem type $\times$ pressure: $F(2,46)=3.560, p=.037, \eta_{\mathrm{p}}^{2}=.134$. When viewing Figure $3 \mathrm{~b}$, it is important to note that the pressure manipulation was always on the second day of testing; the main effect of pressure may be masked by practice effects. Thus, the comparison of the two groups is crucial.

As predicted, pressure had no impact on either group for no carry problems. By contrast, pressure had a differential effect on HWM and LWM participants depending on the specific task in the carry conditions. For normal carry problems, HWM individuals were more affected by pressure than the LWM individuals. That is, HWM individuals

\footnotetext{
${ }^{3}$ Although we did not explicitly include a pressure manipulation check, teachers from the school were confident that those manipulations would place their students under considerable pressure and the first author's observation also confirmed the teachers' confidence (children would sweat and many of them expressed their anxiety to the first author after the test). Furthermore, we note that the pressure manipulation was quite similar to pressure manipulations that have been used in earlier research (Beilock \& Carr, 2005). Finally, the predicted 3-way interaction of pressure, working memory capacity, and problem type is most succinctly explained by an equal effect of pressure in the LWM and HWM groups; a differential effect of pressure on the working memory groups would be difficult to explain. We thank one reviewer of this article, for suggesting the second and third points made here.
} 


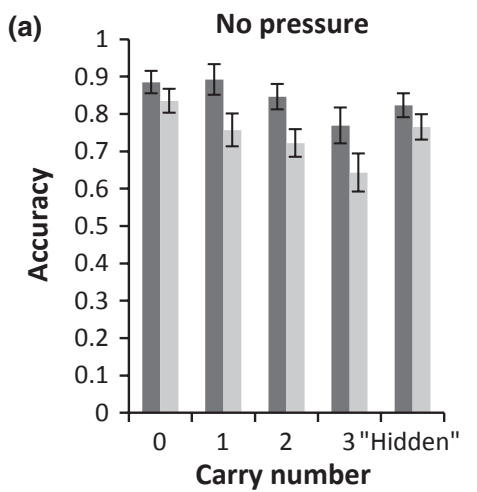

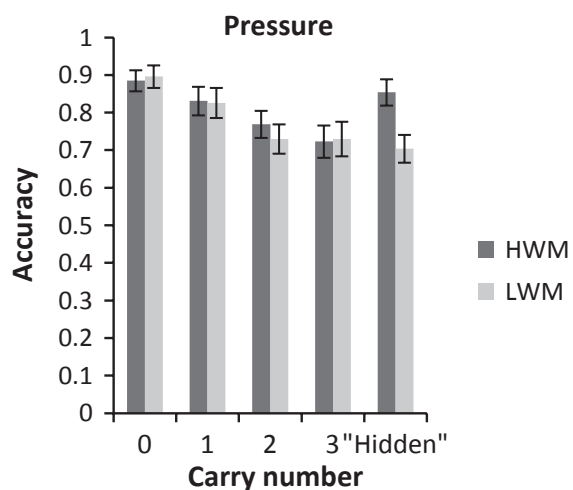

(b) No carry

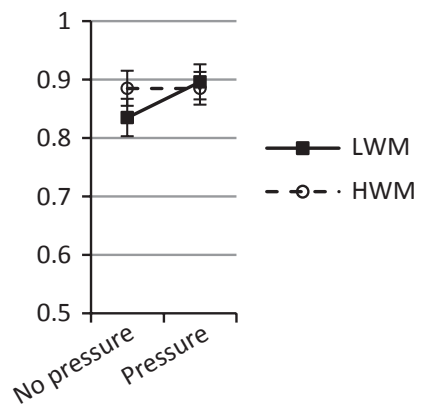

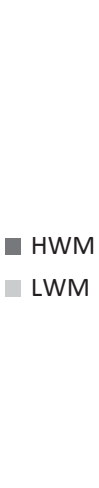

Normal carry

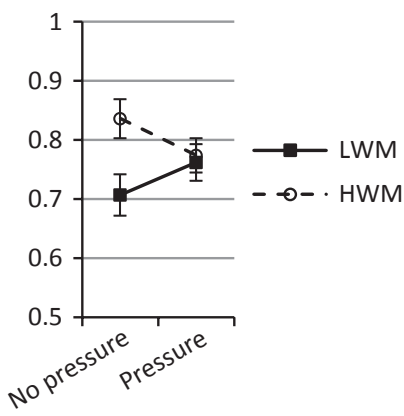

Hidden carry

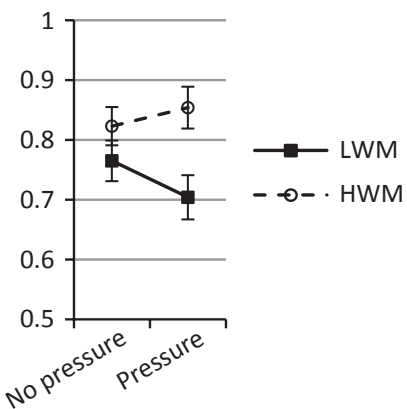

Figure 3. (a) Accuracy data for all the problems divided by carry number. All error bars represent standard error. From no pressure to pressure condition, the difference between high and low working memory (LWM) children decreased except for the hidden carry problems, where the difference was magnified. (b) Accuracy data of different problem types for the two groups in both pressure and no-pressure condition.

performed worse under pressure, whereas LWM did not. Specifically, for these, the two-way interaction was significant, $F(1,47)=4.07, p<.05, \eta_{\mathrm{p}}^{2}=.080$, explained by the fact that when no-pressure HWM participants accuracy was significantly higher than LWMs', $t(47)=2.678, p=.01$, Cohen's $d=.76$; but the difference became non-significant under pressure, $t(47)=0.283, p=.779$, Cohen's $d=.08$.

For the hidden carry problems, although LWM individuals and HWM individuals showed no difference in the no-pressure situation, $t(47)=1.226, p=.226$, Cohen's $d=.35$, LWM individuals performed much worse than HWMs when both of the groups were tested under pressure, $t(47)=2.949, p=.005$, Cohen's $d=.83$. There is a marginal interaction between WM and pressure for these problems, $F(1,47)=2.603, p=.113$, $\eta_{\mathrm{p}}^{2}=.052$.

To investigate the mechanism of the main three-way interaction, we also evaluated the response time data. Figure 4 presents the response time for HWM and LWM children under different conditions. In hidden carry problems, HWM children were consistently faster than LWM children. This is in obvious contrast to normal carry problems, where HWM children under no pressure spent a little longer on the problems but became much faster than LWM children when under pressure. For HWM children, this increase in speed co-occurred with an increase in error rate, indicating that they may have switched to a 
faster but less accurate strategy - the partial calculation strategy. This strategy change in HWM was confirmed by a marginally significant three-way interaction in response time: $F(1,47)=3.923, p=.05, \eta_{\mathrm{p}}^{2}=.077$.

\section{Discussion}

This study replicated previous findings that pressure affects performance on mathematics problem-solving in a new real-life classroom context and helped reconcile contradictory findings of earlier studies on the effects of pressure on cognitive task performance. Some previous research found that pressure decreased the performance difference between HWMs and LWMs (Beilock \& Carr, 2005; Gimmig et al., 2006), whereas other previous research found that pressure magnified HWMs' advantage (Ashcraft \& Kirk, 2001; Ashcraft \& Krause, 2007; Johnson \& Gronlund, 2009). In this study, we reconciled the contradictory findings by exploring the nature of the tasks. We replicated HWMs' choking under pressure with the normal carry task and LWMs' choking under pressure with the hidden carry task. Neither group choked in the easiest no carry task. Our three hypotheses are confirmed: In the no carry (easy) condition, pressure barely affected HWM or LWM children's performance; in the normal carry condition, HWM and LWM children's performance difference disappeared under pressure; in the hidden carry condition, pressure led to the performance difference between HWM children and LWM children.

How pressure affects individual differences in a task depends on the features of the task. The mental arithmetic problems in our study allowed two possible strategies. One is to perform complete calculation; this is more WM demanding but also more accurate, compared with the other strategy, where subjects perform partial calculation and compare the partial results with the two options. Beilock and DeCaro (2007) reported their finding that under pressure, HWM individuals tended to use less-working-memory-intensive strategies. We further explored this finding by considering task features. In our normal carry problems, HWM children were no longer better than LWM under pressure and their reaction time became shorter (Figure 4), indicating that they seemed to have switched to the less accurate but faster partial calculation strategy, which is
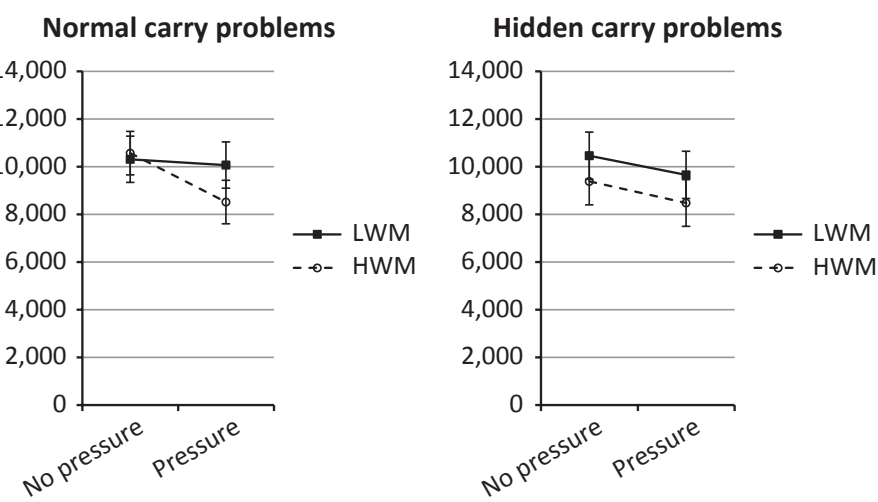

Figure 4. The different RT time patterns in the two kinds of problems indicate a strategy switch in high working memories (HWMs): for the normal carry problems, under pressure HWMs' response became faster, resulting a marginally significant three-way interaction. 
consistent with Beilock and DeCaro (2007) finding. However, in our hidden carry problems, HWM children did not switch to the less-working-memory-intensive strategy, because the use of partial calculation would have significantly hampered performance on these problems.

Based on our results, we argue that the decision of using working-memory-intensive strategies or easier heuristics to solve problems is a complex choice dependent on task-difficulty and a subjects' available working memory. When one's working memory capacity is adequate for the more working memory demanding strategy, subjects will use it. This leads to greater accuracy. However, when an individual's working memory is no longer enough to support the working memory demanding strategy - say, their working memory is consumed by the presence of pressure - they are only able to use heuristic strategies (in this case, calculating only some columns and relying on the choices available to make a decision).

This study makes several contributions to the literature on the effect of pressure on performance. For the first time, we replicated the choking under pressure phenomenon in an elementary school classroom environment. The tasks used in previous studies (such as modular arithmetic and tone discrimination) were relatively unfamiliar to participants. We do not know whether the findings from those studies would also apply to situations outside of the laboratory. In our study, we asked primary school children to perform a mental arithmetic task, which is commonly practised in their daily study, and replicated the choking under pressure phenomenon. The results of our study suggest that in real life, pressure has a differential effect on people with different cognitive abilities; moreover, the differential effect of pressure depends on task demands and their cognitive capacity.

Second, we found that pressure impacts children's test performance from a very young age and in a different culture; previous studies of pressure have used adult and/or college student samples in the West. The differential effects of pressure on high and low WM students could have a substantial long-term effect in the school selection system in China. For a student in China who wants to make it to one of the few key universities around the country (likely a high WM student), she needs to succeed on numerous important tests all through her school years. In the high stake tests such as high school entrance examination and college entrance examination, students suffer from great pressure, knowing that the test score is the only criterion the schools rely on for admission. In all these tests, pressure has a differential effect on different subgroups of students. A variation of test item features in these tests could result in different students getting the opportunity to receive limited higher education resources. Although the participants in this study are Chinese students, we note that the effect of mathematics anxiety appears to have similar effects on achievement in China (mainland) and the United States as well as Taiwan (Ho et al., 2000). Thus, these results are likely relevant for performance in other countries.

More generally, the interaction effect we found among problem type, pressure, and WM has direct implications for educational testing. For institutions that are developing tests, they need to be aware that pressure could magnify or minimize differences in highand low-ability students depending on the nature of problems used. Tasks that do not allow reverting to less intensive WM strategies may be particularly useful if the goal is to identify high-ability students. Given the differential effect of pressure on students with higher or lower capabilities, it may also be important to reduce the pressure conditions. Rather than one high stakes test, for example, children could be evaluated based on numerous tests taken during the school year in more familiar contexts. Another solution 
might be to teach students strategies, such as by talking aloud while they perform mathematics problem-solving tasks, to reduce the impact of pressure on performance (DeCaro, Rotar, Kendra, \& Beilock, 2010).

The methodology and context of this study, although it has several advantages for understanding the effect of pressure in the classroom, in a new cultural context, and for different problem types, has some limitations. These include a relatively small sample size, no direct assessments of strategy use, a fixed order in which the no-pressure context is always first, the use of combined use of multiple types of pressure that may differentially impact performance (DeCaro, Thomas, Albert, \& Beilock, 2011), and no explicit manipulation check. Nonetheless, we clearly find that 'choking under pressure' happens to both HWM and LWM children. Who get more affected by pressure depends on the relative WM requirements of a task. Pressure decreases individual's performance when the task is highly WM demanding for the individual and the individual uses a problem-solving strategy that is working memory intensive.

\section{Acknowledgements}

The authors thank Yanmei Ma, Weizhen Zhang, Yong Feng (school principals), and all the teachers and students at Muling Shiyan Elementary School who made the data collection possible in this study. We also thank the editor and reviewers (one of whom was Sian Beilock) for their helpful comments on this article.

\section{References}

Ashcraft, M. H., \& Kirk, E. P. (2001). The relationships among working memory, math anxiety, and performance. Journal of Experimental Psychology. General, 130, 224-237. doi:10.1037/ 0096-3445.130.2.224

Ashcraft, M. H., \& Krause, J. A. (2007). Working memory, math performance, and math anxiety. Psychonomic Bulletin \& Review, 14, 243-248. doi:10.3758/BF03194059

Baumeister, R. F. (1984). Choking under pressure - Self-consciousness and paradoxical effects of incentives on skillful performance. Journal of Personality and Social Psychology, 46, 610-620. doi:10.1037//0022-3514.46.3.610

Beilock, S. L. (2008). Math performance in stressful situations. Current Directions in Psychological Science, 17, 339-343. doi:10.1111/j.1467-8721.2008.00602.x

Beilock, S. L., \& Carr, T. H. (2005). When high-powered people fail-Working memory and "choking under pressure" in math. Psychological Science, 16, 101-105. doi:10.1111/j.0956-7976.2005. 00789.x

Beilock, S. L., \& DeCaro, M. S. (2007). From poor performance to success under stress: Working memory, strategy selection, and mathematical problem solving under pressure. Journal of Experimental Psychology. Learning, Memory, and Cognition, 33, 983-998. doi:10.1037/ 0278-7393.33.6.983

DeCaro, M. S., Rotar, K. E., Kendra, M. S., \& Beilock, S. L. (2010). Diagnosing and alleviating the impact of performance pressure on mathematical problem solving. Quarterly Journal of Experimental Psychology, 63, 1619-1630. doi:10.1080/17470210903474286

DeCaro, M. S., Thomas, R. D., Albert, N. B., \& Beilock, S. L. (2011). Choking under pressure: Multiple routes to skill failure. Journal of Experimental Psychology: General, 140, 390-406. doi:10.1037/a0023466

Gimmig, D., Huguet, P., Caverni, J. P., \& Cury, F. (2006). Choking under pressure and wording memory capacity: When performance pressure reduces fluid intelligence. Psychonomic Bulletin \& Review, 13, 1005-1010. doi:10.3758/bf03213916 
Hitch, G. J. (1978). Role of short-term working memory in mental arithmetic. Cognitive Psychology, $10,302-323$.

Ho, H.-Z., Senturk, D., Lam, A. G., Zimmer, J. M., Hong, S., Okamoto, Y., ... Wang, C.-P. (2000). The affective and cognitive dimensions of math anxiety: A cross-national study. Journal for Research in Mathematics Education, 31, 362. doi:10.2307/749811

Johnson, D. R., \& Gronlund, S. D. (2009). Individuals lower in working memory capacity are particularly vulnerable to anxiety's disruptive effect on performance. Anxiety, Stress, and Coping, 22, 201-213. doi:10.1080/10615800802291277

Jonides, J. (1995). Working memory and thinking. In E. E. Smith \& D. N. Osherson (Eds.), Thinking: An invitation to cognitive science (Vol. 3, pp. 215-266). Cambridge, MA: MIT Press.

Jonides, J., Lewis, R. L., Nee, D. E., Lustig, C., Berman, M. G., \& Moore, K. S. (2008). The mind and brain of short-term memory. Annual Review of Psychology, 59, 193-224. doi:10.1146/annurev. psych.59.103006.093615

Shah, P., \& Miyake, A. (1999). Models of working memory: Mechanisms of active maintenance and executive control. Cambridge, UK; New York, NY: Cambridge University Press.

Stigler, J. W. (1984). "Mental abacus": The effect of abacus training on Chinese children's mental calculation. Cognitive Psychology, 16, 145-176. doi:10.1016/0010-0285(84)90006-9

Unsworth, N., Heitz, R. R., Schrock, J. C., \& Engle, R. W. (2005). An automated version of the operation span task. Behavior Research Methods, 37, 498-505. doi:10.3758/BF03192720

Received 24 January 2013; revised version received 10 June 2013 